\title{
Impact of material thicknesses on fission observables obtained with the FALSTAFF experimental setup
}

\author{
L. Thulliez ${ }^{1, a}$, D. Doré ${ }^{1}$, E. Berthoumieux ${ }^{1}$, S. Panebianco ${ }^{1}$, P. Legou ${ }^{1}$, M. Kebbiri ${ }^{1}$, Y. Piret ${ }^{1}$, J.P. Mols ${ }^{1}$, \\ M. Combet ${ }^{1}$, M. Riallot ${ }^{1}$, A. Marcel ${ }^{1}$, F. Farget ${ }^{2}$, J. Pancin ${ }^{2}$, M.O. Frégeau ${ }^{2}$, X. Ledoux ${ }^{2}$, F.-R. Lecolley ${ }^{3}$, \\ J. Perronnel ${ }^{3}$, D. Goupillère ${ }^{3}$, and S. Oberstedt ${ }^{4}$ \\ ${ }^{1}$ Irfu, CEA, Université Paris-Saclay, 91191 Gif-sur-Yvette, France \\ 2 GANIL, 14050 Caen, France \\ 3 LPC, 14076 Caen, France \\ ${ }^{4}$ European Commision, DG Joint Research Center, Directorate G - Nuclear Safety and Security, Unit G.2 Standards for Safety, \\ Security and Safeguards, 2440 Geel, Belgium
}

\begin{abstract}
In the past years, the fission studies have been mainly focused on thermal fission because most of the current nuclear reactors work in this energy domain. With the development of GEN-IV reactor concepts, mainly working in the fast energy domain, new nuclear data are needed.

The FALSTAFF spectrometer under development at CEA-Saclay, France, is a two-arm spectrometer which will provide mass yields before ( $2 \mathrm{~V}$ method) and after (EV method) neutron evaporation and consequently will have access to the neutron multiplicity as a function of mass. The axial ionization chamber, in addition to the kinetic energy value, will measure the energy loss profile of the fragment along its track. This energy loss profile will give information about the fragment nuclear charge. This paper will focus on recent developments on the FALSTAFF design. A special attention will be paid to the impact of the detector material thickness on the uncertainty of different observables.
\end{abstract}

\section{Introduction}

Most of the innovative nuclear reactors under development are based on fast reactor technologies. In this fast energy domain, from hundreds $\mathrm{keV}$ to several $\mathrm{MeV}$, new accurate fission fragment mass yields are needed in order to predict the residual decay heat and the poisoning of the nuclear fuel which are important parameters for nuclear reactor management. Experiments are also needed to answer some theoretical questions on the fission process such as how the energy is shared between the two fission fragments. Actually it has been unexpectedly observed that the heavy fragments take the additionnal energy brought by the fast neutron, leading to an enhancement of prompt neutron emission from the heavy fragment while the neutron emission from the light fragments remains the same [1,2].

To answer these questions, a spectrometer named FALSTAFF is under development at CEA-Saclay (France) [3]. The aim of the FALSTAFF spectrometer is to study neutron-induced fission in a neutron energy range from hundreds $\mathrm{keV}$ to several $\mathrm{MeV}$. This energy range will be accessible in installing the spectrometer at the Neutrons For Science (NFS) facility [4]. The advantage of the direct kinematic technique is an accurate definition of the the initial state of the compound nucleus which undergoes fission. However, fission fragments have a low kinetic energy. As a consequence they loose a non-negligible amount of energy when they interact with materials

a e-mail: loic.thulliez@cea.fr making their identification difficult. To correctly identify the fission fragments, the energy losses suffered by the fragments have to be taken into account. This requires the knowledge of the fragment nuclear charge and the material thicknesses crossed by the fragment.

The first part of this paper will describe the setup and the observables accessible with the FALSTAFF spectrometer. The second part will focus on the impact of the detector material thickness uncertainties on the observables. The third part will deal with the impact on the observables, of using thinner materials.

\section{Description of the FALSTAFF spectrometer}

The FALSTAFF spectrometer will provide the full characterization of the fission fragments, i.e. their masses before and after neutron evaporation process, their kinetic energies and their nuclear charges. The deduced neutron multiplicity as a function of mass will provide information on the energy sharing between the two fragments at the scission point.

The mass before neutron evaporation is obtained via the $2 \mathrm{~V}$ (Double Velocity) method. To apply this method the assumption is made that the neutron emission, in average, does not change the velocity of the fragments in the center of mass frame. It requires the measurement of both fragment velocities in coincidence. The velocity is determined with two time-of-flight (ToF) Secondary

(C) The Authors, published by EDP Sciences. This is an Open Access article distributed under the terms of the Creative Commons Attribution License 4.0 (http://creativecommons.org/licenses/by/4.0/). 


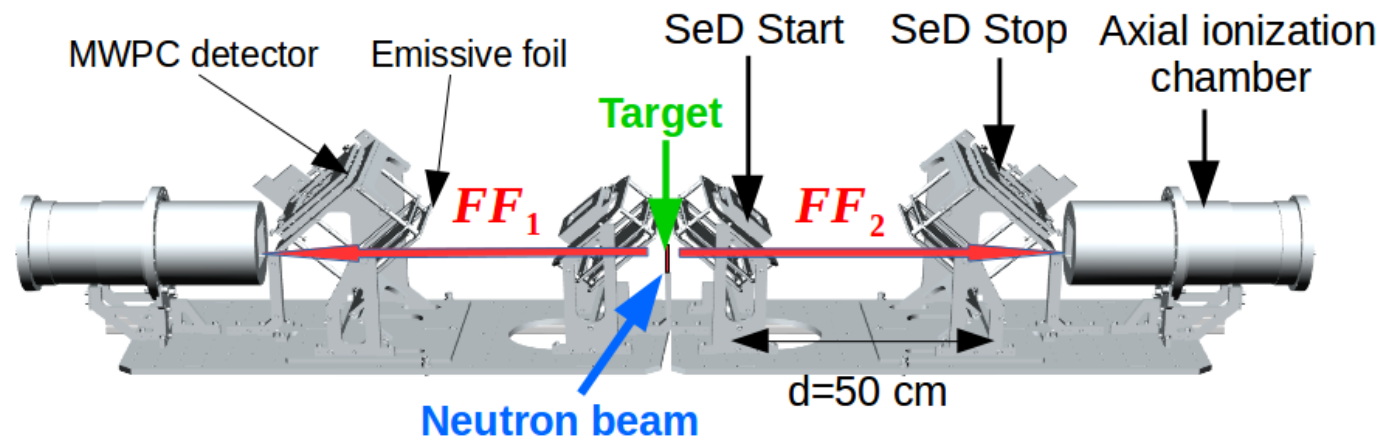

Figure 1. Drawing of the two-arm FALSTAFF spectrometer. Each arm is made of two ToF Secondary Electron Detectors (SeD) and one axial ionization chamber.

Electron Detectors (SeD) [5], represented in Fig. 1. Each detector gives the arrival time and position of a particle on the detector. Those detectors have a timing resolution of $\sigma_{t} \sim 120 \mathrm{ps}$ and a spatial resolution of $\sigma_{X} \sim 1 \mathrm{~mm} \mathrm{[6].}$ The distance between the two SeDs is $50 \mathrm{~cm}$. A SeD is made of an emissive foil and a Multi-Wire Proportional Chamber (MWPC) detector. When a fragment crosses an emissive foil, it looses kinetic energy leading to electron production on the foil surface. The electrons, thanks to an electric field, are then accelerated and detected by the MWPC detector.

The mass after neutron evaporation is obtained with the EV (Energy-Velocity) method. In addition to the velocity information, the kinetic energy value of the fragment is then required. This information is obtained with an axial ionization chamber, CALIBER, placed after the Stop detector. The kinetic energy value measured in the chamber has to be corrected for energy losses suffered by the fragment in the target, in the emissive foils and in the chamber entrance window. Those corrections require the knowledge of the fragment nuclear charge and the thicknesses of the materials the fragments have passed through. The crossed thickness is deduced from the thickness given by the manufacturer and the angle between the detector and the particle trajectory. The nuclear charge information is provided by the energy loss of the fragment along its track.

\section{Impact of the material thickness uncertainty on fission observables}

This section investigates whether or not the average mylar thickness given by the manufacturer can be taken for granted in order to correctly determine the observables and looks at the impact of the thickness uncertainty given by the manufacturer on the observables. The impact of the thickness inhomogeneities on the observable determination is also investigated.

\subsection{Impact of the thickness uncertainty given by the manufacturer}

The emissive foils of the ToF detectors are made of mylar foil having a thickness, given by the manufacturer, of $0.5 \mu \mathrm{m} \pm 20 \%$ and a thin evaporated Aluminium layer of around $30 \mathrm{~nm} \pm 4 \mathrm{~nm}$. The following is dedicated to the investigation of the impact of the thickness uncertainty of the mylar foil on the observables. It is performed in three steps:

a) A Geant-4 [7] geometry of the FALSTAFF spectrometer is implemented in setting the SeD foil thicknesses to $\mathrm{dx}=0.5 \mu \mathrm{m}$. The simulation is performed with the software version Geant4.10.02.p01 and with the Physics-List "QGSP_ BERT_ EMV", from which the fragment energy losses depend.

b) Fission events, from the spontaneous fission of ${ }^{252} \mathrm{Cf}$, from GEF code [8] are then generated. The software version 2015/2.2 (01/2016) is used. The number of neutron emitted by each fragment is provided by this software, leading to the neutron multiplicity as a function of mass represented by the red curve in Fig. 2. It shows a plateau around masses 140-155, perhaps due to too high shell corrections.

c) The simulated data collected in the detectors are analysed under different assumptions on the foil thicknesses.

In the following, the impact of the hypothesis is investigated by looking at their effects on the neutron multiplicity as a function of mass.

For this study the analysis is performed with different assumptions: emissive foils have a thickness of 1) $\mathrm{dx}=0.5 \mu \mathrm{m}$ (red curve), 2$) \mathrm{dx}=0.4 \mu \mathrm{m}$ (green curve) and 3) $\mathrm{dx}=0.6 \mu \mathrm{m}$ (blue curve) representing respectively the lower limit $-20 \%$ and the upper limit of $+20 \%$. The results are shown in Fig. 2. The hypothesis 1) corresponds to the case where the material thickness is fully known and thus can be considered as the reference. The difference between green and blue curves represents the neutron multiplicity uncertainty due to the thickness uncertainty. This amplitude range goes from 1 to 4 neutrons for the light fragments and from 4 to 6 neutrons for the heavy ones. The neutron uncertainty is bigger for heavy fragments than for light ones because they suffer more energy losses, so more energy loss corrections have to be applied. The blue result in Fig. 2 presents a negative neutron multiplicity associated to the heavy fragments. This is due to an overestimation of the foil thickness compared to the real one. In fact, this overestimation leads to too large energy loss corrections, leading to too large final masses. It does not affect initial masses which are obtained via the $2 \mathrm{~V}$ method for which less energy loss corrections are needed.

The results show that the average thickness value given by the manufacturer can not be taken for granted because 


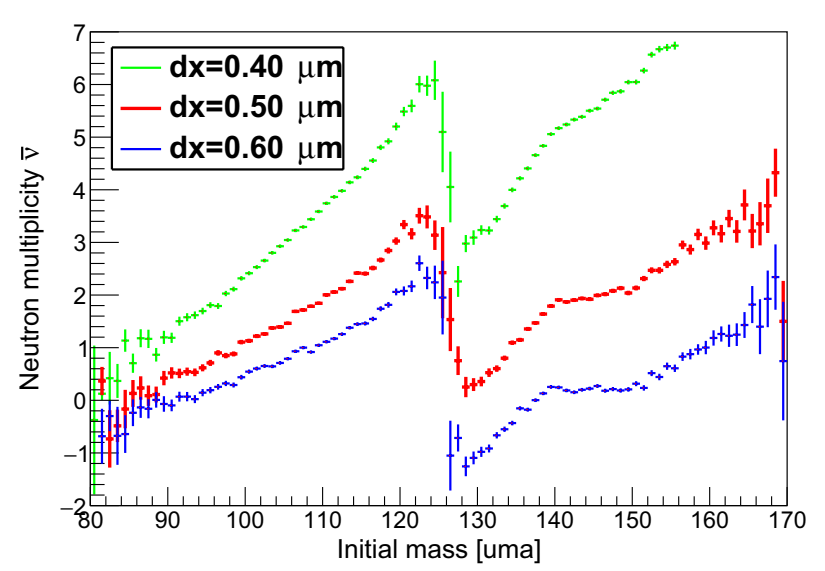

Figure 2. Impact of the thickness uncertainty given by the manufacturer on the neutron multiplicity as a function of mass from the reaction ${ }^{252} \mathrm{Cf}(\mathrm{sf})$.

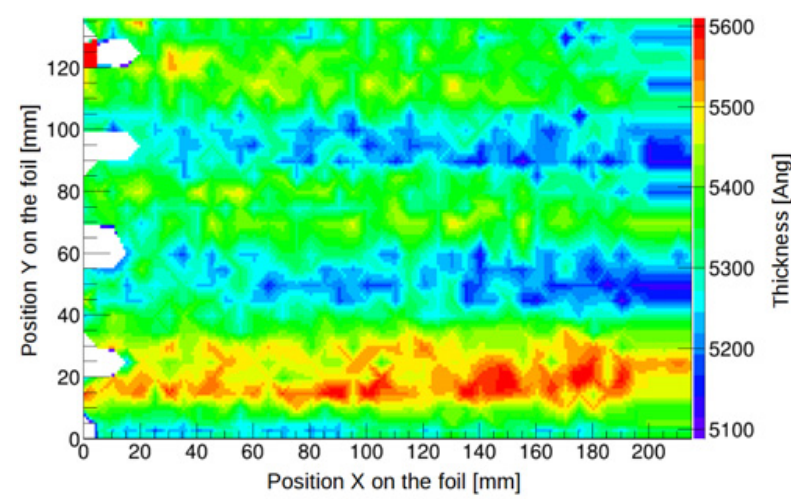

Figure 3. A typical foil thickness profile obtained after thickness measurements based on the energy loss of alpha-particles from ${ }^{241} \mathrm{Am}$.

of the high impact of the thickness uncertainty given by the manufacturer on the observables.

\subsection{Thickness measurement setup and thickness profile reconstruction method}

To reduce the thickness uncertainty given by the manufacturer, a thickness measurement setup has been developed based on the $\alpha$-transmission technique. The detection setup is made of an ${ }^{241} \mathrm{Am}$ source and a PIPS Silicon detector which has an energy resolution of $12 \mathrm{keV}$ [9], leading to a measurement thickness precison of $\pm 10 \mathrm{~nm}$. The energy loss of alpha particles through a foil is measured and then converted in mylar thickness with the help of energy loss/thickness table, built with the SRIM software [10]. A scan of the $14 \times 22 \mathrm{~cm}^{2}$ foil is performed and then the entire foil profile is reconstructed with a Delaunay triangulation interpolation [11]. A typical thickness profile is presented in Fig. 3. The visible structures depend on the way the mylar foil is stretched and sticked to its frame.

\subsection{Impact of the thickness inhomogeneity}

Once the foil profile is obtained, the impact of the thickness inhomogeneity of the foil on the observable is studied. The spatial resolution of the thickness measurement setup is equal to $5 \mathrm{~mm}$. This determines the minimum size of the measurement step. The foil profile

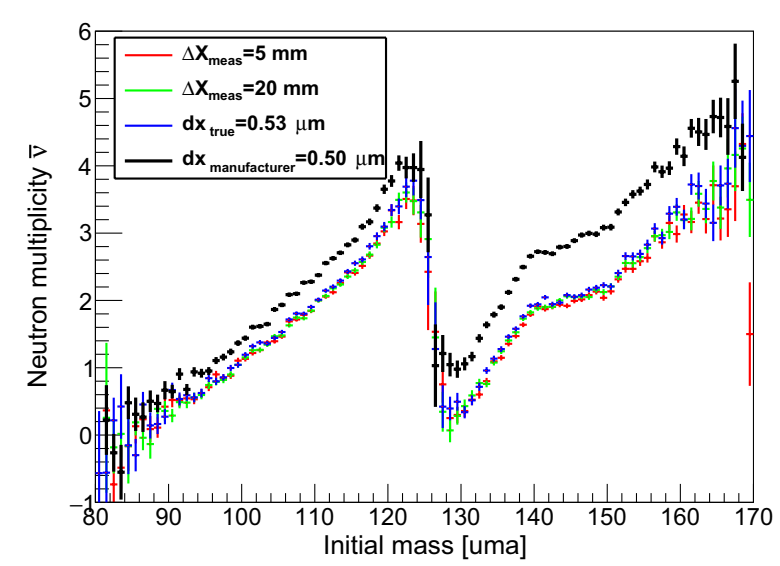

Figure 4. Impact of the measurement step on the neutron multiplicity as a function of mass.

reconstructed from measurements performed every $5 \mathrm{~mm}$, represented in Fig. 3, is implemented in Geant-4. Fission events are then generated.

The analysis of the simulated data collected by the detectors are then performed with different assumptions. The neutron multiplicity as a function of initial mass, represented in Fig. 4, are obtained according to the following hypothesis:

1) a complete knowledge of the profile, red results.

2) a partial knowledge of the profile (basically one point out of four is kept from measurements performed every $5 \mathrm{~mm}$ ), green results.

3) an average thickness of $0.53 \mu \mathrm{m}$, blue results.

4) an average thickness of $0.5 \mu \mathrm{m}$, black results.

The similarities of those results, depicted in Fig. 4 by red $(\Delta X=5 \mathrm{~mm})$ and green $(\Delta X=20 \mathrm{~mm})$ symbols, show that the determination of the fission observables does not depend on the measurement step. Moreover only the knowledge of the true average thickness is necessary (blue line). This true average thickness is defined as the mean of the measured thicknesses from the profil in Fig. 3. Thus measurements have to be performed to find the true average thickness. In this case, the measured average thickness is $0.53 \mu \mathrm{m}$, which is different from the one given by the manufacturer $(0.5 \mu \mathrm{m})$. So far the measured average thickness does not depend on the measurement step $(5 \mathrm{~mm}$ or $20 \mathrm{~mm}$ ). Special attention is needed in the accuracy of this statement since only few foils have been characterized.

\section{Impact of the foil thickness on velocity observable}

The main difficulty of studying fission in direct kinematic is the particle identification due to energy losses in the different detectors. Previously the study of inhomogeneity impact was also performed for a $0.9 \mu \mathrm{m}$ thick emissive foil. The conclusions are the same as those drawn for the $0.5 \mu \mathrm{m}$ thick foil. As a consequence, to minimize the impact of the energy loss correction and its uncertainty, the thinner foil is used.

As shown in Fig. 5, the measured velocity, determined by fitting each peak by a gaussian, is then shifted up by $\sim 0.04 \mathrm{~cm} / \mathrm{ns}$ for the light and heavy fragments when a foil of $0.5 \mu \mathrm{m}$ is used instead of $0.9 \mu \mathrm{m}$ one. This corresponds approximately to an energy loss reduction 


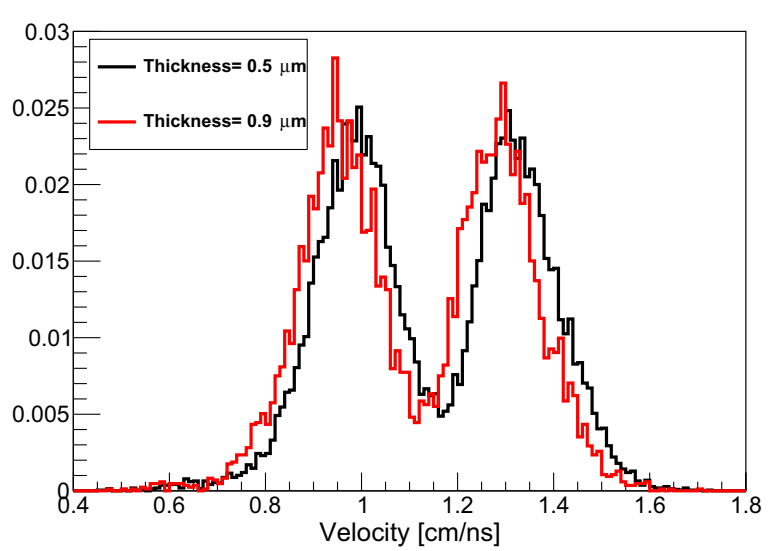

Figure 5. Comparison of velocity measurements performed with emissive foils made from mylar of thickness $0.5 \mu \mathrm{m}$ (black line) and $0.9 \mu \mathrm{m}$ (red line).

of around $\sim 10 \mathrm{MeV}$ (Geant-4 results). As a consequence the kinetic energy measured value in the axial ionization chamber with a foil of $0.5 \mu \mathrm{m}$ is higher than with the one of $0.9 \mu \mathrm{m}$. This also leads to a longer energy loss profile measurement, which consequently brings more information for the fragment nuclear charge determination.

\section{Conclusion}

One key point in the studies of neutron-induced fission is to take correctly into account the energy losses suffered by the fission fragments in the detector materials in order to correctly identify the fragments. The present study has shown the non-negligible impact of the thickness uncertainty given by the manufacturer on the observables. As a consequence a thickness measurement setup has been built to determine the thickness profile of the SeD emissive foils and of the ionization chamber entrance window. This measured average thickness might be different from the average thickness given by the manufacturer and has to be known. It has been pointed out that taking into account the thickness inhomogeneities is not necessary, and that only the measured average foil thickness is needed. It was also shown that using a foil of $0.5 \mu \mathrm{m}$ instead of the original $0.9 \mu \mathrm{m}$ one, has been successfully conducted leading to smaller energy loss corrections. Usage of foil thinner than $0.9 \mu \mathrm{m}$ was a technical challenge in itself.

The next step for the development of the FALSTAFF spectrometer is to characterize the new axial ionization chamber, CALIBER. Its characterization will be made with a ${ }^{252} \mathrm{Cf}$ source and its energy calibration will be made with ion beams. A method for the fragment nuclear charge determination from the fragment energy loss profile in CALIBER will be investigated. The full characterization of the first arm of the spectrometer will be done in 2017 and the two-arm FALSTAFF spectrometer will be ready for data taking in 2018.

This work was supported by EURATOM 7 Framework Programme CHANDA and the French programme NEEDS.

\section{References}

[1] A.A. Naqvi et al., Phys. Rev. C 34, 218 (1986)

[2] R. Müller et al., Phys. Rev. C 29, 885 (1984)

[3] D. Doré et al., Nuclear Data Sheets 119, 346 (2014)

[4] X. Ledoux et al., Nuclear Data Sheets 119, 353 (2014)

[5] A. Drouart et al., Nucl. Instr. Meth. Phys. Res. A 477, 401 (2002)

[6] M. Vostinar et al., JINST 119 (2013)

[7] S. Agostinelli et al., Nucl. Instr. Meth. Phys. Res. A 506, 250 (2003)

[8] K.H. Schmidt et al., Tech. rep., JEFF Report 24 (2014)

[9] http://www. canberra.com/products/ detectors/pips-detectors-standard.asp, accessed: 01/04/2014

[10] J. Ziegler, http://www.srim.org, accessed: $05 / 2014$

[11] P. Lancaster and K. Salkauskas, Curve and Surface Fitting, an introduction, 3rd edn. (Academic Press, 1990) 